\title{
Split general quasi-variational inequality problem
}

\author{
K.R. Kazmil \\ Department of Mathematics, Aligarh Muslim University, Aligarh-202002, India
}

\begin{abstract}
In this paper, we introduce a split general quasi-variational inequality problem which is a natural extension of split variational inequality problem, quasivariational and variational inequality problems in Hilbert spaces. Using projection method, we propose an iterative algorithm for the split general quasi-variational inequality problem and discuss some its special cases. Further, we discuss the convergence criteria of these iterative algorithms. The results presented in this paper generalize, unify and improve the previously known many results for the quasi-variational and variational inequality problems.

Keywords: Split general quasi-variational inequality problem, Split quasi-variational inequality problem, Split general variational inequality problem, iterative algorithms, convergence analysis.
\end{abstract}

AMS Subject Classifications: Primary 47J53, 65K10; Secondary 49M37, 90C25.

\section{Introduction}

Throughout the paper unless otherwise stated, for each $i \in\{1,2\}$, let $H_{i}$ be a real Hilbert space with inner product $\langle\cdot, \cdot\rangle$ and norm $\|\cdot\|$; let $C_{i}$ be a nonempty, closed and convex subset of $H_{i}$.

The variational inequality problem (in short, VIP) is to find $x_{1} \in C_{1}$ such that

$$
\left\langle f_{1}\left(x_{1}\right), y_{1}-x_{1}\right\rangle \geq 0, \quad \forall y_{1} \in C_{1}
$$

where $f_{1}: C_{1} \rightarrow H_{1}$ be a nonlinear mapping.

Variational inequality theory introduced by Stampacchia [1] and Fichera [2] independently, in early sixties in potential theory and mechanics, respectively, constitutes a significant extension of variational principles. It has been shown that the variational inequality theory provides the natural, descent, unified and efficient framework for a

\footnotetext{
${ }^{1}$ emails: kr.kazmi.mm@amu.ac.in; krkazmi@gmail.com (K.R. Kazmi)
} 
general treatment of a wide class of unrelated linear and nonlinear problem arising in elasticity, economics, transportations, optimization, control theory and engineering sciences, see for instance [3-8]. The development of variational inequality theory can be viewed as the simultaneous pursuit of two different lines of research. On the one hand, it reveals the fundamental facts on the qualitative behavior of solutions to important classes of problems. On the other hands, it enables us to develop highly efficient and powerful numerical methods to solve, for example, obstacle, unilateral, free and moving boundary value problems. In last five decades, considerable interest has been shown in developing various classes of variational inequality problems, both for its own sake and for its applications.

An important generalization of variational inequality problem is quasi-variational inequality problem introduced and studied by Bensoussan, Goursat and Lions [9] in connection of impulse control problems. More presicely, for each $i$, let $C_{i}: H_{i} \rightarrow 2^{H_{i}}$ be a nonempty, closed and convex set valued mapping, where $2^{H_{i}}$ be the family of all nonempty subsets of $H_{i}$. The quasi-variational inequality problem (in short, QVIP) is to find $x_{1} \in H_{1}$ such that $x_{1} \in C_{1}\left(x_{1}\right)$ and

$$
\left\langle f_{1}\left(x_{1}\right), y_{1}-x_{1}\right\rangle \geq 0, \quad \forall y_{1} \in C_{1}\left(x_{1}\right),
$$

where $f_{1}: H_{1} \rightarrow H_{1}$ be a nonlinear mapping.

We observe that if $C_{1}\left(x_{1}\right)=C_{1}$ for all $x_{1} \in H_{1}$, then $\operatorname{QVIP}(1.2)$ is reduced to $\operatorname{VIP}(1.1)$. In many important applications, $C_{1}\left(x_{1}\right)=m\left(x_{1}\right)+C_{1}$ for each $x_{1} \in H_{1}$, where $m: H_{1} \rightarrow H_{1}$ is a single valued mapping, see for instance [4,5]. Since then various generalization of $\operatorname{QVIP}(1.2)$ have been proposed and analyzed, see for instance [10-14].

Recently, Censor et al. [15] introduced and studied the following split variational inequality problem (in short, SpVIP): For each $i \in\{1,2\}$, let $f_{i}: H_{i} \rightarrow H_{i}$ be a nonlinear mapping and $A: H_{1} \rightarrow H_{2}$ be a bounded linear operator with its adjoint operator $A^{*}$. Then the SpVIP is to:

Find $x_{1}^{*} \in C_{1}$ such that

$$
\left\langle f_{1}\left(x_{1}^{*}\right), x_{1}-x_{1}^{*}\right\rangle \geq 0, \quad \forall x_{1} \in C_{1},
$$

and such that

$$
x_{2}^{*}=A x_{1}^{*} \in C_{2} \text { solves }\left\langle f_{2}\left(x_{2}^{*}\right), x_{2}-x_{2}^{*}\right\rangle \geq 0, \quad \forall x_{2} \in C_{2} .
$$


$\operatorname{SpVIP}(1.3 \mathrm{a}-\mathrm{b})$ amount to saying: find a solution of variational inequality problem $\operatorname{VIP}(1.3 \mathrm{a})$, the image of which under a given bounded linear operator is a solution of VIP(1.3b). It is worth mentioning that SpVIP is quite general and permits split minimization between two spaces so the image of a minimizer of a given function, under a bounded linear operator, is a minimizer of another function. $\operatorname{SpVIP}(1.3 \mathrm{a}-\mathrm{b})$ is an important generalization of $\operatorname{VIP}(1.1)$. It also includes as special case, the split zero problem and split feasibility problem which has already been studied and used in practice as a model in intensity-modulated radiation therapy treatment planning, see [16-18]. For the further related work, we refer to see Moudafi [19], Byrne et al. [20], Kazmi and Rizvi [21-24] and Kazmi [25].

In this paper, we introduce the following natural generalization of $\operatorname{SpVIP}(1.3 \mathrm{a}-\mathrm{b})$ : For each $i \in\{1,2\}$, let $C_{i}: H_{i} \rightarrow 2^{H_{i}}$ be a nonempty, closed and convex set valued mapping. For each $i \in\{1,2\}$, let $f_{i}: H_{i} \rightarrow H_{i}$ and $g_{i}: H_{i} \rightarrow H_{i}$ be nonlinear mappings and $A: H_{1} \rightarrow H_{2}$ be a bounded linear operator with its adjoint operator $A^{*}$. Then we consider the problem:

Find $x_{1}^{*} \in H_{1}$ such that $g_{1}\left(x_{1}^{*}\right) \in C_{1}\left(x_{1}^{*}\right)$ and

$$
\left\langle f_{1}\left(x_{1}^{*}\right), x_{1}-g_{1}\left(x_{1}^{*}\right)\right\rangle \geq 0, \quad \forall x_{1} \in C_{1}\left(x_{1}^{*}\right)
$$

and such that

$$
x_{2}^{*}=A x_{1}^{*} \in H_{2}, \quad g_{2}\left(x_{2}^{*}\right) \in C_{2}\left(x_{2}^{*}\right) \text { solves }\left\langle f_{2}\left(x_{2}^{*}\right), x_{2}-g_{2}\left(x_{2}^{*}\right)\right\rangle \geq 0, \quad \forall x_{2} \in C_{2}\left(x_{2}^{*}\right) .
$$

We call the problem (1.4a-b), the split general quasi-variational inequality problem (in short, SpGQVIP).

Now, we observe some special cases of SpGQVIP(1.4a-b).

If we set $g_{i}=I_{i}$, where $I_{i}$ is identity operator on $H_{i}$, then $\operatorname{SpGQVIP}(1.4 \mathrm{a}-\mathrm{b})$ is reduced to the following split quasi-variational inequality problem (in short, SpQVIP):

Find $x_{1}^{*} \in H_{1}$ such that $x_{1}^{*} \in C_{1}\left(x_{1}^{*}\right)$ and

$$
\left\langle f_{1}\left(x_{1}^{*}\right), x_{1}-x_{1}^{*}\right\rangle \geq 0, \quad \forall x_{1} \in C_{1}\left(x_{1}^{*}\right),
$$

and such that

$$
x_{2}^{*}=A x_{1}^{*} \in H_{2}, x_{2}^{*} \in C_{2}\left(x_{2}^{*}\right) \text { solves }\left\langle f_{2}\left(x_{2}^{*}\right), x_{2}-x_{2}^{*}\right\rangle \geq 0, \quad \forall x_{2} \in C_{2}\left(x_{2}^{*}\right),
$$


which appears to be new.

If we set $C_{i}\left(x_{i}\right)=C_{i}$ for all $x_{i} \in H_{i}$, then $\operatorname{SpGQVIP}(1.4 \mathrm{a}-\mathrm{b})$ is reduced to following split general variational inequality problem (in short, SpGVIP):

Find $x_{1}^{*} \in H_{1}$ such that $g_{1}\left(x_{1}^{*}\right) \in C_{1}$ and

$$
\left\langle f_{1}\left(x_{1}^{*}\right), x_{1}-g_{1}\left(x_{1}^{*}\right)\right\rangle \geq 0, \quad \forall x_{1} \in C_{1},
$$

and such that

$$
x_{2}^{*}=A x_{1}^{*} \in H_{2}, \quad g_{2}\left(x_{2}^{*}\right) \in C_{2} \text { solves }\left\langle f_{2}\left(x_{2}^{*}\right), x_{2}-g_{2}\left(x_{2}^{*}\right)\right\rangle \geq 0, \quad \forall x_{2} \in C_{2},
$$

which appears to be new.

Further, if we set $C_{i}\left(x_{i}\right)=C_{i}$ for all $x_{i} \in H_{i}$, and $g_{i}=I_{i}$, then $\operatorname{SpGQVIP(1.4a-b)}$ is reduced to $\operatorname{SpVIP}(1.3 \mathrm{a}-\mathrm{b})$.

Furthermore, if we set $H_{2}=H_{1} ; C_{2}\left(x_{2}\right)=C_{1}\left(x_{1}\right) \forall x_{i} ; f_{2}=f_{1}$, and $g_{i}=I_{i}$, then $\operatorname{SpGQVIP}(1.4 \mathrm{a}-\mathrm{b})$ is reduced to $\operatorname{QVIP}(1.2)$.

Using projection method, we propose an iterative algorithm for SpGQVIP(1.4a-b) and discuss some its special cases which are the iterative algorithms for SpQVIP(1.5ab), SpGVIP(1.6a-b), SpVIP(1.3a-b) and QVIP(1.2). Further, we discuss the convergence criteria of these iterative algorithms. The results presented in this paper generalize, unify and improve the previously known many results for the quasi-variational and variational inequality problems.

\section{Iterative algorithms}

For each $i \in\{1,2\}$, a mapping $P_{C_{i}}$ is said to be metric projection of $H_{i}$ onto $C_{i}$ if for every point $x_{i} \in H_{i}$, there exists a unique nearest point in $C_{i}$ denoted by $P_{C_{i}}\left(x_{i}\right)$ such that

$$
\left\|x_{i}-P_{C_{i}}\left(x_{i}\right)\right\| \leq\left\|x_{i}-\bar{x}_{i}\right\|, \quad \forall \bar{x}_{i} \in C_{i}
$$

It is well known that $P_{C_{i}}$ is nonexpansive mapping and satisfies

$$
\left\langle x_{i}-\bar{x}_{i}, P_{C_{i}}\left(x_{i}\right)-P_{C_{i}}\left(\bar{x}_{i}\right)\right\rangle \geq\left\|P_{C_{i}}\left(x_{i}\right)-P_{C_{i}}\left(\bar{x}_{i}\right)\right\|^{2}, \quad \forall x_{i}, \bar{x}_{i} \in H_{i} .
$$

Moreover, $P_{C_{i}}\left(x_{i}\right)$ is characterized by:

$$
\left\langle x_{i}-P_{C_{i}}\left(x_{i}\right), \bar{x}_{i}-P_{C_{i}}\left(x_{i}\right)\right\rangle \leq 0, \quad \forall \bar{x}_{i} \in C_{i}
$$


Further, it is easy to see that the following is true:

$x_{1}^{*}$ is a solution of $\operatorname{QVIP}(1.2) \Leftrightarrow x_{1}^{*}=P_{C_{1}\left(x_{1}^{*}\right)}\left(x_{1}^{*}-\rho_{1} f_{1}\left(x_{1}^{*}\right)\right), \quad \rho_{1}>0$.

Hence, SpGQVIP(1.4a-b) can be reformulated as follows: Find $x_{1}^{*} \in H_{1}$ with $x_{2}^{*}=A x_{1}^{*}$ such that $g_{i}\left(x_{i}^{*}\right) \in C_{i}\left(x_{i}^{*}\right)$ and

$$
g_{i}\left(x_{i}^{*}\right)=P_{C_{i}\left(x_{i}^{*}\right)}\left(g_{i}\left(x_{i}^{*}\right)-\rho_{i} f_{i}\left(x_{i}^{*}\right)\right)
$$

for $\rho_{i}>0$.

Based on above arguments, we propose the following iterative algorithm for approximating a solution to $\operatorname{SpGQVIP}(1.4 \mathrm{a}-\mathrm{b})$.

Let $\left\{\alpha^{n}\right\} \subseteq(0,1)$ be a sequence such that $\sum_{n=1}^{\infty} \alpha^{n}=+\infty$, and let $\rho_{1}, \rho_{2}, \gamma$ are parameters with positive values.

Iterative algorithm 2.1. Given $x_{1}^{0} \in H_{1}$, compute the iterative sequences $\left\{x_{1}^{n}\right\}$ defined by the iterative schemes:

$$
\begin{gathered}
g_{1}\left(y^{n}\right)=P_{C_{1}\left(x_{1}^{n}\right)}\left(g_{1}\left(x_{1}^{n}\right)-\rho_{1} f_{1}\left(x_{1}^{n}\right)\right) \\
g_{2}\left(z^{n}\right)=P_{C_{2}\left(A y^{n}\right)}\left(g_{2}\left(A y^{n}\right)-\rho_{2} f_{2}\left(A y^{n}\right)\right) \\
x_{1}^{n+1}=\left(1-\alpha^{n}\right) x_{1}^{n}+\alpha^{n}\left[y^{n}+\gamma A^{*}\left(z^{n}-A y^{n}\right)\right]
\end{gathered}
$$

for all $n=0,1,2, \ldots ., \rho_{i}, \gamma>0$.

If we set $g_{i}=I_{i}$, then Iterative algorithm 2.1 is reduced to following iterative algorithm for $\operatorname{SpQVIP}(1.5 \mathrm{a}-\mathrm{b})$ :

Iterative algorithm 2.2. Given $x_{1}^{0} \in H_{1}$, compute the iterative sequences $\left\{x_{1}^{n}\right\}$ defined by the iterative schemes:

$$
\begin{gathered}
y^{n}=P_{C_{1}\left(x_{1}^{n}\right)}\left(x_{1}^{n}-\rho_{1} f_{1}\left(x_{1}^{n}\right)\right) \\
z^{n}=P_{C_{2}\left(A y^{n}\right)}\left(A y^{n}-\rho_{2} f_{2}\left(A y^{n}\right)\right) \\
x_{1}^{n+1}=\left(1-\alpha^{n}\right) x_{1}^{n}+\alpha^{n}\left[y^{n}+\gamma A^{*}\left(z^{n}-A y^{n}\right)\right]
\end{gathered}
$$

for all $n=0,1,2, \ldots . ., \rho_{i}, \gamma>0$. 
If we set $C_{i}\left(x_{i}\right)=C_{i}$ for all $x_{i} \in H_{i}$, then Iterative algorithm 2.1 is reduced to following iterative algorithm for $\operatorname{SpGVIP(1.6a-b):~}$

Iterative algorithm 2.3. Given $x_{1}^{0} \in H_{1}$, compute the iterative sequences $\left\{x_{1}^{n}\right\}$ defined by the iterative schemes:

$$
\begin{gathered}
g_{1}\left(y^{n}\right)=P_{C_{1}}\left(g_{1}\left(x_{1}^{n}\right)-\rho_{1} f_{1}\left(x_{1}^{n}\right)\right) \\
g_{2}\left(z^{n}\right)=P_{C_{2}}\left(g_{2}\left(A y^{n}\right)-\rho_{2} f_{2}\left(A y^{n}\right)\right) \\
x_{1}^{n+1}=\left(1-\alpha^{n}\right) x_{1}^{n}+\alpha^{n}\left[y^{n}+\gamma A^{*}\left(z^{n}-A y^{n}\right)\right]
\end{gathered}
$$

for all $n=0,1,2, \ldots ., \rho_{i}, \gamma>0$.

If we set $C_{i}\left(x_{i}\right)=C_{i}$ for all $x_{i} \in H_{i}$, and $g_{i}=I_{i}$, then Iterative algorithm 2.1 is

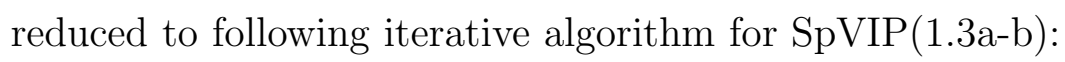

Iterative algorithm 2.4[25]. Given $x_{1}^{0} \in H_{1}$, compute the iterative sequences $\left\{x_{1}^{n}\right\}$ defined by the iterative schemes:

$$
\begin{gathered}
y^{n}=P_{C_{1}}\left(x_{1}^{n}-\rho_{1} f_{1}\left(x_{1}^{n}\right)\right) \\
z^{n}=P_{C_{2}}\left(A y^{n}-\rho_{2} f_{2}\left(A y^{n}\right)\right) \\
x_{1}^{n+1}=\left(1-\alpha^{n}\right) x_{1}^{n}+\alpha^{n}\left[y^{n}+\gamma A^{*}\left(z^{n}-A y^{n}\right)\right]
\end{gathered}
$$

for all $n=0,1,2, \ldots . ., \rho_{i}, \gamma>0$.

If we set $H_{2}=H_{1} ; C_{2}\left(x_{2}\right)=C_{1}\left(x_{1}\right) \forall x_{i} ; f_{2}=f_{1}$, and $g_{i}=I_{i}$, then Iterative algorithm 2.1 is reduced to following Mann iterative algorithm for QVIP(1.2):

Iterative algorithm 2.5. Given $x_{1}^{0} \in H_{1}$, compute the iterative sequences $\left\{x_{1}^{n}\right\}$ defined by the iterative schemes:

$$
\begin{gathered}
y^{n}=P_{C_{1}}\left(x_{1}^{n}-\rho_{1} f_{1}\left(x_{1}^{n}\right)\right) \\
x_{1}^{n+1}=\left(1-\alpha^{n}\right) x_{1}^{n}+\alpha^{n} y^{n}
\end{gathered}
$$

for all $n=0,1,2, \ldots . ., \rho_{1}>0$.

Assumption 2.1. For all $x_{i}, y_{i}, z_{i} \in H_{i}$, the operator $P_{C_{i}\left(x_{i}\right)}$ satisfies the condition:

$$
\left\|P_{C_{i}\left(x_{i}\right)}\left(z_{i}\right)-P_{C_{i}\left(y_{i}\right)}\left(z_{i}\right)\right\| \leq \nu_{i}\left\|x_{i}-y_{i}\right\|,
$$

for some constant $\nu_{i}>0$.

Definition 2.1. A nonlinear mapping $f_{1}: H_{1} \rightarrow H_{1}$ is said to be: 
(i) $\alpha_{1}$-strongly monotone, if there exists a constant $\alpha_{1}>0$ such that

$$
\left\langle f_{1}(x)-f_{1}(\bar{x}), x-\bar{x}\right\rangle \geq \alpha\|x-\bar{x}\|^{2}, \quad \forall x, \bar{x} \in H_{1}
$$

(ii) $\beta_{1}$-Lipschitz continuous, if there exists a constant $\beta_{1}>0$ such that

$$
\left\|f_{1}(x)-f_{1}(\bar{x})\right\| \leq \beta\|x-\bar{x}\|, \quad \forall x, \bar{x} \in H_{1} .
$$

\section{Results}

Now, we study the convergence of the Iterative algorithm 2.1 for SpGQVIP(1.4ab).

Theorem 3.1. For each $i \in\{1,2\}$, let $C_{i}: H_{i} \rightarrow 2^{H_{i}}$ be a nonempty, closed and convex set valued mapping. Let $f_{i}: H_{i} \rightarrow H_{i}$ be $\alpha_{i}$-strongly monotone with respect to $g_{i}$ and $\beta_{i}$-Lipschitz continuous and let $g_{i}: H_{i} \rightarrow H_{i}$ be $\delta_{i}$-Lipschitz continuous and $\left(g_{i}-I_{i}\right)$ be $\sigma_{i}$-strongly monotone, where $I_{i}$ is the identity operator on $H_{i}$. Let $A: H_{1} \rightarrow H_{2}$ be a bounded linear operator and $A^{*}$ be its adjoint operator. Suppose $x_{1}^{*} \in H_{1}$ is a solution to $\operatorname{SpGQVIP}(1.4 \mathrm{a}-\mathrm{b})$ and Assumption 2.1 hold, then the sequence $\left\{x_{1}^{n}\right\}$ generated by Iterative algorithm 2.1 converges strongly to $x_{1}^{*}$ provided that the constants $\rho_{i}$ and $\gamma$ satisfy the following conditions:

$$
\begin{gathered}
\left|\rho_{1}-\frac{\alpha_{1}}{\beta_{1}^{2}}\right|<\frac{\sqrt{\alpha_{1}^{2}-\beta_{1}^{2}\left(\delta_{1}^{2}-k_{1}^{2}\right)}}{\beta_{1}^{2}} \\
\alpha_{1}>\beta_{1} \sqrt{\delta_{1}^{2}-k_{1}^{2}} ; \quad k_{1}=\left[\frac{\sqrt{2 \sigma_{1}+1}}{1+2 \theta_{2}}-\nu_{1}\right] ; \quad \delta_{1}>\left|k_{1}\right| ; \\
0<\theta_{2}=\frac{1}{\sqrt{2 \sigma_{2}+1}}\left\{\sqrt{\delta_{2}^{2}-2 \rho_{2} \alpha_{2}+\rho_{2}^{2} \beta_{2}^{2}}+\nu_{2}\right\} ; \quad \rho_{2}>0 ; \quad \gamma \in\left(0, \frac{2}{\|A\|^{2}}\right)
\end{gathered}
$$

Proof. Since $x_{1}^{*} \in H_{1}$ is a solution to $\operatorname{SpGQVIP}(1.4 \mathrm{a}-\mathrm{b})$, then $x_{1}^{*} \in H_{1}$ be such that $g_{i}\left(x_{i}^{*}\right) \in C_{i}\left(x_{i}^{*}\right)$ and

$$
\begin{gathered}
g_{1}\left(x_{1}^{*}\right)=P_{C_{1}\left(x_{1}^{*}\right)}\left(g_{1}\left(x_{1}^{*}\right)-\rho_{1} f_{1}\left(x_{1}^{*}\right)\right), \\
g_{2}\left(A x_{1}^{*}\right)=P_{C_{2}\left(A x_{1}^{*}\right)}\left(g_{2}\left(A x_{1}^{*}\right)-\rho_{2} f_{2}\left(A x_{1}^{*}\right)\right),
\end{gathered}
$$

for $\rho_{i}>0$.

From Iterative algorithm 2.1(2.4a), Assumption 2.1 and (3.1), we have

$$
\left\|g_{1}\left(y^{n}\right)-g_{1}\left(x_{1}^{*}\right)\right\|=\left\|P_{C_{1}\left(x_{1}^{n}\right)}\left(g_{1}\left(x_{1}^{n}\right)-\rho_{1} f_{1}\left(x_{1}^{n}\right)\right)-P_{C_{1}\left(x_{1}^{*}\right)}\left(g_{1}\left(x_{1}^{*}\right)-\rho_{1} f_{1}\left(x_{1}^{*}\right)\right)\right\|
$$




$$
\begin{aligned}
& \leq\left\|P_{C_{1}\left(x_{1}^{n}\right)}\left(g_{1}\left(x_{1}^{n}\right)-\rho_{1} f_{1}\left(x_{1}^{n}\right)\right)-P_{C_{1}\left(x_{1}^{n}\right)}\left(g_{1}\left(x_{1}^{*}\right)-\rho_{1} f_{1}\left(x_{1}^{*}\right)\right)\right\| \\
& +\left\|P_{C_{1}\left(x_{1}^{n}\right)}\left(g_{1}\left(x_{1}^{*}\right)-\rho_{1} f_{1}\left(x_{1}^{*}\right)\right)-P_{C_{1}\left(x_{1}^{*}\right)}\left(g_{1}\left(x_{1}^{*}\right)-\rho_{1} f_{1}\left(x_{1}^{*}\right)\right)\right\| \\
& \leq\left\|g_{1}\left(x_{1}^{n}\right)-g_{1}\left(x_{1}^{*}\right)-\rho_{1}\left(f_{1}\left(x_{1}^{n}\right)-f_{1}\left(x_{1}^{*}\right)\right)\right\|+\nu_{1}\left\|x_{1}^{n}-x_{1}^{*}\right\| .
\end{aligned}
$$

Now, using the fact that $f_{1}$ is $\alpha_{1}$-strongly monotone with respect to $g_{1}$ and $\beta_{1^{-}}$ Lipschitz continuous, and $g_{1}$ is $\delta_{1}$-Lipschitz continuous, we have

$$
\begin{aligned}
\| g_{1}\left(x_{1}^{n}\right)- & g_{1}\left(x_{1}^{*}\right)-\rho_{1}\left(f_{1}\left(x_{1}^{n}\right)-f_{1}\left(x_{1}^{*}\right)\right) \|^{2} \\
= & \left\|g_{1}\left(x_{1}^{n}\right)-g_{1}\left(x_{1}^{*}\right)\right\|^{2}-2 \rho_{1}\left\langle f_{1}\left(x_{1}^{n}\right)-f_{1}\left(x_{1}^{*}\right), g_{1}\left(x_{1}^{n}\right)-g_{1}\left(x_{1}^{*}\right)\right\rangle \\
& +\rho^{2}\left\|f_{1}\left(x_{1}^{n}\right)-f_{1}\left(x_{1}^{*}\right)\right\|^{2} \\
& \leq\left(\delta_{1}^{2}-2 \rho_{1} \alpha_{1}+\rho_{1}^{2} \beta_{1}^{2}\right)\left\|x_{1}^{n}-x_{1}^{*}\right\|^{2} .
\end{aligned}
$$

As a result, we obtain

$$
\left\|g_{1}\left(y^{n}\right)-g_{1}\left(x_{1}^{*}\right)\right\| \leq\left\{\sqrt{\delta_{1}^{2}-2 \rho_{1} \alpha_{1}+\rho_{1}^{2} \beta_{1}^{2}}+\nu_{1}\right\}\left\|x_{1}^{n}-x_{1}^{*}\right\| .
$$

Since $\left(g_{1}-I_{1}\right)$ is $\sigma_{1}$-strongly monotone, we have

$$
\begin{aligned}
\left\|y^{n}-x_{1}^{*}\right\|^{2} \leq & \left\|g_{1}\left(y^{n}\right)-g_{1}\left(x_{1}^{*}\right)\right\|^{2}-2\left\langle\left(g_{1}-I_{1}\right) y^{n}-\left(g_{1}-I_{1}\right) x_{1}^{*}, y^{n}-x_{1}^{*}\right\rangle \\
& \leq\left\|g_{1}\left(y^{n}\right)-g_{1}\left(x_{1}^{*}\right)\right\|^{2}-2 \sigma_{1}\left\|y^{n}-x_{1}^{*}\right\|^{2},
\end{aligned}
$$

which implies

$$
\left\|y^{n}-x_{1}^{*}\right\| \leq \frac{1}{\sqrt{2 \sigma_{1}+1}}\left\|g_{1}\left(y^{n}\right)-g_{1}\left(x_{1}^{*}\right)\right\|
$$

From (3.3) and (3.4), we have

$$
\left\|y^{n}-x_{1}^{*}\right\| \leq \theta_{1}\left\|x_{1}^{n}-x_{1}^{*}\right\|,
$$

where $\theta_{1}=\frac{1}{\sqrt{2 \sigma_{1}+1}}\left\{\sqrt{\delta_{1}^{2}-2 \rho_{1} \alpha_{1}+\rho_{1}^{2} \beta_{1}^{2}}+\nu_{1}\right\}$.

Similarly, from Iterative algorithm 2.1(2.4b), Assumption 2.1 and (3.2) and using the fact that $f_{2}$ is $\alpha_{2}$-strongly monotone with respect to $g_{2}$ and $\beta_{2}$-Lipschitz continuous, and $\left(g_{2}-I_{2}\right)$ is $\sigma_{2}$-strongly monotone, and $g_{2}$ is $\delta_{2}$-Lipschitz continuous, we have

$$
\left\|g_{2}\left(z^{n}\right)-g_{2}\left(A x_{1}^{*}\right)\right\| \leq\left\{\sqrt{\delta_{2}^{2}-2 \rho_{2} \alpha_{2}+\rho_{2}^{2} \beta_{2}^{2}}+\nu_{2}\right\}\left\|A y^{n}-A x_{1}^{*}\right\|,
$$


and

$$
\left\|z^{n}-A x_{1}^{*}\right\| \leq \theta_{2}\left\|A y^{n}-A x_{1}^{*}\right\|,
$$

where $\theta_{2}=\frac{1}{\sqrt{2 \sigma_{2}+1}}\left\{\sqrt{\delta_{2}-2 \rho_{2} \alpha_{2}+\rho_{2}^{2} \beta_{2}^{2}}+\nu_{2}\right\}$.

Next, from Iterative algorithm 2.1(2.4c), we have

$\left\|x_{1}^{n+1}-x_{1}^{*}\right\| \leq\left(1-\alpha^{n}\right)\left\|x_{1}^{n}-x_{1}^{*}\right\|+\alpha^{n}\left[\left\|y^{n}-x_{1}^{*}-\gamma A^{*}\left(A y^{n}-A x_{1}^{*}\right)\right\|+\gamma\left\|A^{*}\left(z^{n}-A x_{1}^{*}\right)\right\|\right]$

Further, using the definition of $A^{*}$, fact that $A^{*}$ is a bounded linear operator with $\left\|A^{*}\right\|=\|A\|$, and given condition on $\gamma$, we have

$$
\begin{gathered}
\left\|y^{n}-x_{1}^{*}-\gamma A^{*}\left(A y^{n}-A x_{1}^{*}\right)\right\|^{2}=\left\|y^{n}-x_{1}^{*}\right\|^{2}-2 \gamma\left\langle y^{n}-x_{1}^{*}, A^{*}\left(A y^{n}-A x_{1}^{*}\right)\right\rangle+\gamma^{2}\left\|A^{*}\left(A y^{n}-A x_{1}^{*}\right)\right\|^{2} \\
\leq\left\|y^{n}-x_{1}^{*}\right\|^{2}-\gamma\left(2-\gamma\|A\|^{2}\right)\left\|A y^{n}-A x_{1}^{*}\right\|^{2} \\
\leq\left\|y^{n}-x_{1}^{*}\right\|^{2}
\end{gathered}
$$

and, using (3.8), we have

$$
\begin{aligned}
\left\|A^{*}\left(z^{n}-A x_{1}^{*}\right)\right\| & \leq\|A\|\left\|z^{n}-A x_{1}^{*}\right\| \\
& \leq \theta_{2}\|A\|\left\|A y^{n}-A x_{1}^{*}\right\| \\
& \leq \theta_{2}\|A\|^{2}\left\|y^{n}-x_{1}^{*}\right\| .
\end{aligned}
$$

Combining (3.10) and (3.11) with inequality (3.9), as a result, we obtain

$$
\left\|x_{1}^{n+1}-x_{1}^{*}\right\| \leq\left[1-\alpha^{n}(1-\theta)\right]\left\|x_{1}^{n}-x_{1}^{*}\right\|,
$$

where $\theta=\theta_{1}\left(1+\gamma\|A\|^{2} \theta_{2}\right)$.

Hence, after $n$ iterations, we obtain

$$
\left\|x_{n+1}-x^{*}\right\| \leq \prod_{j=1}^{n}\left[1-\alpha_{j}(1-\theta)\right]\left\|x_{0}-x^{*}\right\| .
$$

It follows from conditions on $\rho_{1} \rho_{2}$ that $\theta \in(0,1)$. Since $\sum_{n=1}^{\infty} \alpha^{n}=+\infty$ and $\theta \in(0,1)$, it implies in the light of $[10]$ that

$$
\lim _{n \rightarrow \infty} \prod_{j=1}^{n}\left[1-\alpha_{j}(1-\theta)\right]=0 .
$$


Thus it follows from (3.12) that $\left\{x_{n}\right\}$ converges strongly to $x^{*}$ as $n \rightarrow+\infty$. Since $A$ is continuous, it follows from (3.3),(3.6), (3.7) and (3.8) that $y^{n} \rightarrow x_{1}^{*}, g_{1}\left(y^{n}\right) \rightarrow g_{1}\left(x_{1}^{*}\right)$ $A y^{n} \rightarrow A x_{1}^{*}, z^{n} \rightarrow A x_{1}^{*}$ and $g_{2}\left(z^{n}\right) \rightarrow g_{2}\left(A x_{1}^{*}\right)$ as $n \rightarrow+\infty$. This completes the proof.

If we set $g_{i}=I_{i}$, then Theorem 3.1 reduces to the following result for the convergence of the Iterative algorithm 2.2 for $\operatorname{SpQVIP}(1.5 \mathrm{a}-\mathrm{b})$.

Corollary 3.1. For each $i \in\{1,2\}$, let $C_{i}: H_{i} \rightarrow 2^{H_{i}}$ be a nonempty, closed and convex set valued mapping. Let $f_{i}: H_{i} \rightarrow H_{i}$ be $\alpha_{i}$-strongly monotone and $\beta_{i}$-Lipschitz continuous and let $A: H_{1} \rightarrow H_{2}$ be a bounded linear operator and $A^{*}$ be its adjoint operator. Suppose $x_{1}^{*} \in H_{1}$ is a solution to $\operatorname{SpQVIP}(1.5 \mathrm{a}-\mathrm{b})$ and Assumption 2.1 hold, then the sequence $\left\{x_{1}^{n}\right\}$ generated by Iterative algorithm 2.2 converges strongly to $x_{1}^{*}$ provided that the constants $\rho_{i}$ and $\gamma$ satisfy the following conditions:

$$
\begin{gathered}
\left|\rho_{1}-\frac{\alpha_{1}}{\beta_{1}^{2}}\right|<\frac{\sqrt{\alpha_{1}^{2}-\beta_{1}^{2}\left(1-k_{1}^{2}\right)}}{\beta_{1}^{2}} \\
\alpha_{1}>\beta_{1} \sqrt{1-k_{1}^{2}} ; \quad k_{1}=\frac{1}{1+2 \theta_{2}}-\nu_{1} ; \quad\left|k_{1}\right|<1 ; \\
0<\theta_{2}=\left\{\sqrt{1-2 \rho_{2} \alpha_{2}+\rho_{2}^{2} \beta_{2}^{2}}+\nu_{2}\right\} ; \quad \rho_{2}>0 ; \quad \gamma \in\left(0, \frac{2}{\|A\|^{2}}\right)
\end{gathered}
$$

If we set $C_{i}\left(x_{i}\right)=C_{i}, \forall x_{i} \in H_{i}$ then Theorem 3.1 reduces to the following result for the convergence of the Iterative algorithm 2.3 for $\operatorname{SpGVIP(1.6a-b).~}$

Corollary 3.2. For each $i \in\{1,2\}$, let $C_{i}$ be a nonempty, closed and convex set in $H_{i}$. Let $f_{i}: H_{i} \rightarrow H_{i}$ be $\alpha_{i}$-strongly monotone with respect to $g_{i}$ and $\beta_{i}$-Lipschitz continuous and let $g_{i}: H_{i} \rightarrow H_{i}$ be $\delta_{i}$-Lipschitz continuous and $\left(g_{i}-I_{i}\right)$ be $\sigma_{i}$-strongly monotone, where $I_{i}$ is the identity operator on $H_{i}$. Let $A: H_{1} \rightarrow H_{2}$ be a bounded linear operator and $A^{*}$ be its adjoint operator. Suppose $x_{1}^{*} \in H_{1}$ is a solution to $\operatorname{SpGVIP}(1.6 \mathrm{a}-\mathrm{b})$ and Assumption 2.1 hold, then the sequence $\left\{x_{1}^{n}\right\}$ generated by Iterative algorithm 2.3 converges strongly to $x_{1}^{*}$ provided that the constants $\rho_{i}$ and $\gamma$ satisfy the following conditions:

$$
\begin{gathered}
\left|\rho_{1}-\frac{\alpha_{1}}{\beta_{1}^{2}}\right|<\frac{\sqrt{\alpha_{1}^{2}-\beta_{1}^{2}\left(\delta_{1}^{2}-k_{1}^{2}\right)}}{\beta_{1}^{2}} \\
\alpha_{1}>\beta_{1} \sqrt{\delta_{1}^{2}-k_{1}^{2}} ; \quad k_{1}=\frac{\sqrt{2 \sigma_{1}+1}}{1+2 \theta_{2}} ; \quad \delta_{1}>\left|k_{1}\right| ;
\end{gathered}
$$




$$
0<\theta_{2}=\sqrt{\frac{\delta_{2}^{2}-2 \rho_{2} \alpha_{2}+\rho_{2}^{2} \beta_{2}^{2}}{2 \sigma_{2}+1}} ; \quad \rho_{2}>0 ; \quad \gamma \in\left(0, \frac{2}{\|A\|^{2}}\right)
$$

If we set $H_{2}=H_{1} ; C_{2}\left(x_{2}\right)=C_{1}\left(x_{1}\right) \forall x_{i} ; f_{2}=f_{1} ; A=I_{1}$, and $g_{i}=I_{i}$, then The-

orem 3.1 reduces to the following result for the convergence of the Iterative algorithm 2.5 for $\mathrm{QVIP}(1.2)$.

Corollary 3.3. Let $C_{1}: H_{1} \rightarrow 2^{H_{1}}$ be a nonempty, closed and convex set valued mapping. Let $f_{1}: H_{1} \rightarrow H_{1}$ be $\alpha_{1}$-strongly monotone and $\beta_{1}$-Lipschitz continuous. Suppose $x_{1}^{*} \in H_{1}$ is a solution to QVIP(1.2) and Assumption 2.1 hold, then the sequence $\left\{x_{1}^{n}\right\}$ generated by Iterative algorithm 2.5 converges strongly to $x_{1}^{*}$ provided that the constant $\rho_{1}$ satisfies the following conditions:

$$
\begin{gathered}
\left|\rho_{1}-\frac{\alpha_{1}}{\beta_{1}^{2}}\right|<\frac{\sqrt{\alpha_{1}^{2}-\beta_{1}^{2}\left(1-k_{1}^{2}\right)}}{\beta_{1}^{2}} \\
\alpha_{1}>\beta_{1} \sqrt{1-k_{1}^{2}} ; \quad k_{1}=1-\nu_{1} ; \quad\left|k_{1}\right|<1 .
\end{gathered}
$$

Remark 3.1. It is of further research effort to extend the iterative method presented in this paper for solving the split variational inclusions [19] and the split equilibrium problem [22].

\section{References}

1. Stampacchia, G: Formes bilinearires coercitives sur les ensembles convexes. C.R. Acad. Sci. Paris 258, 4413-4416 (1964)

2. Fichera, G: Problemi elastostatici con vincoli unilaterali: Il problema di Signorini ambigue condizione al contorno. Attem. Acad. Naz. Lincei. Mem. Cl. Sci. Nat. Sez. Ia 7(8), 91-140 (1963/64)

3. Bensoussan, A, Lions, JL: Applications of Variational Inequalities to Stochastic Control. North-Holland, Amsterdam, 1982

4. Bensoussan, A, Lions, JL: Impulse Control and Quasivariational Inequalities. Gauthiers Villers, Paris, 1984.

5. Baiocchi, C, Capelo, A: Variational and Quasi-variational Inequalities. Wiley, New York, 1984 
6. Crank, J: Free and Moving Boundary Problems. Clarendon Press, Oxford, 1984

7. Glowinski, R: Numerical Methods for Nonlinear Variational Problems. Springer, Berlin, 1984

8. Kikuchi, N, Oden, JT: Contact Problems in Elasticity. SIAM, Philadelphia, 1998

9. Bensoussan, A, Goursat,M, Lions, JL: Contrôle impulsinnel et inequations quasivariationnelles stationeries. C.R. Acad. Sci. 276, 1279-1284 (1973)

10. Kazmi, KR: On a class of quasivariational inequalities. New Zealand J. Math. 24, 17-23 (1995)

11. Kazmi, KR: Mann and Ishikawa type perturbed iterative algorithms for generalized quasi-variational inclusions. J. Math. Anal. Appl. 209, 572-584 (1997)

12. Kazmi, KR, Bhat, MI, Khan, FA: A class of multi-valued quasi-variational inequalities. J. Nonlinear Convex Anal. 6(3), 487-495 (2005)

13. Kazmi, KR: Iterative algorithm for a class of generalized quasi-variational inclusions with fuzzy mappings in Banach spaces. J. Comput. Appl. Math. 188(1), $1-11(2006)$

14. Kazmi, KR, Khan, FA, Shahzad, M: Existence and iterative approximation of a unique solution of a system of general quasi-variational inequality problems, Thai J. Math. 8(2), 405-417 (2010)

15. Censor, Y, Gibali, A, Reich, S: Algorithms for the split variational inequality problem. Numerical Algorithms 59, 301-323 (2012)

16. Censor, Y, Bortfeld, T, Martin, B, Trofimov, A: A unified approach for inversion problems in intensity modulated radiation therapy. Physics in Medicine and Biology 51, 2353-2365 (2006)

17. Censor, Y, Elfving, T: A multiprojection algorithm using Bergman projections in product space. Numerical Algorithms 8, 221-239 (1994)

18 Combettes, PL: The convex feasibility problem in image recovery. Adv. Imaging Electron Phys. 95, 155-270 (1996) 
19. Moudafi, A: Split monotone variational inclusions. J. Optim. Theory Appl. 150, 275-283 (2011)

20. Byrne, C, Censor, Y, Gibali, A, Reich, S: Weak and strong convergence of algorithms for the split common null point problem. J. Nonlinear Convex Anal. 13, 759-775 (2012)

21. Kazmi, KR, Rizvi, SH: Iterative approximation of a common solution of a split equilibrium problem, a variational inequality problem and a fixed point problem. J. Egyptian Math. Soc. 21, 44-51 (2013)

22. Kazmi, KR, Rizvi, SH: Iterative approximation of a common solution of a split generalized equilibrium problem and a fixed point problem for nonexpansive semigroup, Mathematical Sciences 7, Art. 1 (2013) (doi 10.1186/2251-7456-7-1)

23. Kazmi, KR, Rizvi, SH: An iterative method for split variational inclusion problem and fixed point problem for a nonexpansive mapping. In Press, Optimization Letters (2013)(doi 10.1007/s11590-013-0629-2)

24. Kazmi, KR, Rizvi, SH: Implicit iterative method for approximating a common solution of split equilibrium problem and fixed point problem for a nonexpansive semigroup. In Press, Arab J. Math. Sci. (2013)(doi 10.1016/j.ajmsc.2013.04.002)

25. Kazmi, KR: Split nonconvex variational inequality problem. Mathematical Sciences 7, Art. 36 (2013) (doi: 10.1186/10.1186/2251-7456-7-36) 